\title{
Approximate Electromagnetic Cloaking of a Dielectric Sphere Using Homogeneous Isotropic Multi-Layered Materials
}

\author{
Hany M. Zamel ${ }^{1}$, Essam El Diwany ${ }^{1}$, Hadia El Hennawy ${ }^{2}$ \\ ${ }^{1}$ Electronics Research Institute (ERI), Microwave Engineering Department, Cairo, Egypt; ${ }^{2}$ Faculty of Engineering, Ain Shams Uni- \\ versity, Cairo, Egypt. \\ Email: res_ass@yahoo.com
}

Received August $6^{\text {th }}, 2013$; revised September $7^{\text {th }}, 2013$; accepted September $28^{\text {th }}, 2013$

Copyright (C) 2013 Hany M. Zamel et al. This is an open access article distributed under the Creative Commons Attribution License, which permits unrestricted use, distribution, and reproduction in any medium, provided the original work is properly cited.

\begin{abstract}
In cloaking, a body is hidden from detection by surrounding it by a coating consisting of an unusual anisotropic nonhomogeneous material. The permittivity and permeability of such a cloak are determined by the coordinate transformation of compressing a hidden body into a point or a line. The radially-dependent spherical cloaking shell can be approximately discretized into many homogeneous anisotropic layers; each anisotropic layer can be replaced by a pair of equivalent isotropic sub-layers, where the effective medium approximation is used to find the parameters of these two equivalent sub-layers. In this work, the scattering properties of cloaked dielectric sphere is investigated using a combination of approximate cloaking, where the dielectric sphere is transformed into a small sphere rather than to a point, together with discretizing the cloaking material using pairs of homogeneous isotropic sub-layers. The back-scattering normalized radar cross section, the scattering patterns are studied and the total scattering cross section against the frequency for different number of layers and transformed radius.
\end{abstract}

Keywords: Approximate Cloaking; Dielectric Sphere; Cloaking by Layered Isotropic Materials

\section{Introduction}

Recently, the concept of electromagnetic cloaking has drawn considerable attention concerning theoretical, numerical and experimental aspects [1-8]. One approach to achieve electromagnetic cloaking is to deflect the rays that would have struck the object, guide them around the object, and return them to their original trajectory, thus no waves are scattered from the body [1]. In the coordinate transformation method for cloaking, the body to be hidden is transformed virtually into a point (3D or spherical configuration) or a line (2D or cylindrical configuration), and this transformation leads to the profile of $\varepsilon, \mu$ in the cloaking coating. Some components of the electrical parameters of the cloaking material $(\varepsilon, \mu)$ are required to have infinite or zero value at the boundary of the hidden object. This requires the use of metamaterials which can produce such values, however, they are narrow band since they rely on using array of resonant elements (as split ring resonators) [9-12].

Approximate cloaking can be achieved by transforming the hidden body virtually into a small object rather than a point or a line as shown in Figure 1, which eliminates the zero (point transformed) or infinite (line transformed) values of the electrical parameters $[13,14]$. This, however, leads to some scattering, since the hidden body is virtually transformed into a small object rather than a point or a line, and the scattering decreases as the transformed sphere radius is smaller.

The radially-dependent spherical cloaking shell can be approximately discretized into many homogeneous anisotropic layers, provided that the thickness of each layer is much less than the wavelength, and this discretization decreases the level of scattering as the number of layers increases. Each anisotropic layer can be replaced by a pair of equivalent isotropic sub-layers, where the effective medium approximation is used to find the parameters of these two equivalent sub-layers $[15,16]$. Near the boundary of the hidden sphere, the values of the radial components of $\varepsilon, \mu$ of the cloaking material are nearly zero. This makes the values of $\varepsilon, \mu$ in one of the pair of isotropic layers to be very small, and can be implemented using metamaterials $[11,17,18]$. Two approaches are 
possible in choosing the material properties in the pair of sub-layers. The first is to take the smaller values of $\varepsilon, \mu$ to be in one layer and the larger values to be in the second layers [19], whereas the other possibility is to take the smaller value of $\varepsilon$ together with the larger value of $\mu$ in one sub-layer, and the opposite combination in the other sub-layer [20]. The scattering properties of cloaked conducting sphere [21] and conducting cylinder [22] were investigated using a combination of approximate cloaking, where the conducting sphere is transformed into a small sphere rather than a point and the conducting cylinder is transformed into small cylinder rather than a line, together with discretizing the cloaking material using pairs of homogeneous isotropic sub-layers.

In this work, the scattering properties of cloaked dielectric sphere is investigated using a combination of approximate cloaking, where the dielectric sphere is transformed into a small sphere rather than a point, together with discretizing the cloaking material using pairs of homogeneous isotropic sub-layers. The solution is obtained by rigorously solving Maxwell equations using Mie series expansion. The back-scattering normalized radar cross section, the scattering cross section patterns are studied and the total scattering cross section against the frequency for different numbers of layers and the transformed radius.

\section{Design Parameters of the Approximate Spherical Cloak}

Perfect spherical cloak can be constructed by compressing the electromagnetic fields in a spherical region $r^{\prime} \leq R_{2}$ into a spherical shell $R_{1} \leq r \leq R_{2}$ as shown in Figure 1. The coordinate transformation is $r^{\prime}=f(r)$, with $f\left(R_{1}\right)=0$ for perfect cloaking or $f\left(R_{1}\right)=c$ for approximate cloaking and $f\left(R_{2}\right)=R_{2}$ [14]. The radial and transverse permittivity and permeability of the spherical cloak, depending on $r$, are given as [23]:

$$
\frac{\varepsilon_{r}}{\varepsilon_{0}}=\frac{\mu_{r}}{\mu_{0}}=\frac{f(r)^{2}}{r^{2} f^{\prime}(r)}, \frac{\varepsilon_{\theta}}{\varepsilon_{0}}=\frac{\mu_{\theta}}{\mu_{0}}=\frac{\varepsilon_{\varnothing}}{\varepsilon_{0}}=\frac{\mu_{\varnothing}}{\mu_{0}}=f^{\prime}(r)
$$

A linear transformation is usually used, given for approximate cloaking by (for ideal cloaking $c=0$ ) [14], [24]:

$$
f(r)=r^{\prime}=\frac{1}{\left(R_{2}-R_{1}\right)}\left[r\left(R_{2}-c\right)+R_{2}\left(c-R_{1}\right)\right]
$$

Thus, the permittivity and permeability of the approximate spherical cloak are given from the above equations by:

$$
\frac{\varepsilon_{r}}{\varepsilon_{0}}=\frac{\mu_{r}}{\mu_{0}}=\frac{\left[r\left(R_{2}-c\right)+R_{2}\left(c-R_{1}\right)\right]^{2}}{r^{2}\left(R_{2}-R_{1}\right)\left(R_{2}-c\right)}
$$

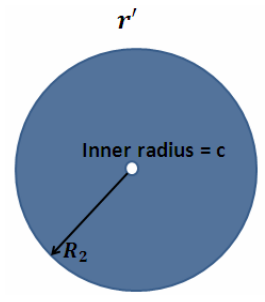

(a)

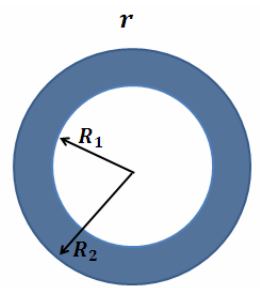

(b)
Figure 1. (a) Virtual domain, (b) Actual domain.

$$
\frac{\varepsilon_{t}}{\varepsilon_{0}}=\frac{\mu_{t}}{\mu_{0}}=\frac{\left(R_{2}-c\right)}{\left(R_{2}-R_{1}\right)}
$$

at $r=R_{1}$,

$$
\frac{\varepsilon_{r}}{\varepsilon_{0}}=\frac{c^{2}\left(R_{2}-R_{1}\right)}{R_{1}^{2}\left(R_{2}-c\right)}
$$

Thus, the permittivity at $r=R_{1}$ decreases as the cloaked radius $c$ and the shell thickness decrease with values less than unity. At $r=R_{2}$,

$$
\frac{\varepsilon_{r}}{\varepsilon_{0}}=\frac{\left(R_{2}-R_{1}\right)}{\left(R_{2}-c\right)}
$$

\section{The Discretization of an Anisotropic Nonhomogeneous Spherical Cloak Using Isotropic Layered Materials}

We first discretize the cloaking anisotropic and nonhomogeneous shell into $2 M$ homogenous layers with identical thickness $\left(d_{B}=d_{A}=d\right)$ as shown in Figure 2, and then the outer radius of each sub-layer is:

$$
r_{i+1}=R_{1}+i \frac{R_{2}-R_{1}}{2 M}, \cdots i=1,2, \cdots, 2 M
$$

An anisotropic layer can be replaced by a pair of equivalent isotropic sub-layers, where the effective medium approximation is used to find the parameters of these two equivalent sub-layers, Figure 2 [19,20]. The radial parameter $\sigma_{r}($ relative $\varepsilon$ or $\mu)$ in the anisotropic layer can be considered as a series combination of the parameters of the equivalent sublayers $\sigma_{1}, \sigma_{2}$ (considered as capacitors) related by:

$$
\frac{1}{\sigma_{r}}=\frac{1}{2 \sigma_{1}}+\frac{1}{2 \sigma_{2}}
$$

The tangential parameters $\sigma_{t}$ in the anisotropic layer can be considered as a parallel combination of the parameters of the equivalent sub-layers $\sigma_{1}, \sigma_{2}$ related by:

$$
\sigma_{t}=\left(\sigma_{1}+\sigma_{2}\right) / 2
$$

One can obtain the equivalent medium parameters for the isotropic sub-layers as: 


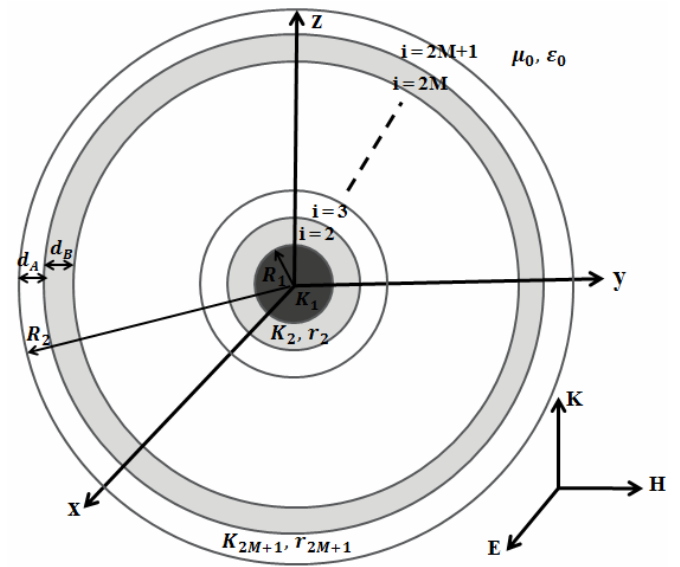

Figure 2. Plane wave scattering by a multi-layer dielectric sphere.

$$
\begin{gathered}
\sigma_{1}=\sigma_{t}+\sqrt{\sigma_{t}^{2}-\sigma_{t} \sigma_{r}} \\
\sigma_{2}=\sigma_{t}-\sqrt{\sigma_{t}^{2}-\sigma_{t} \sigma_{r}}
\end{gathered}
$$

where $\sigma_{r}, \sigma_{t}$ are given by Equations (3) and (4). $\sigma_{2}$ is small, particularly near the conducting sphere where $\sigma_{r}$ is nearly zero. The value of $r$ for a discretized layer is taken at the average radius of this layer i.e. at the interface of layers $\mathrm{A}$ and $\mathrm{B}$.

Two approaches are possible in choosing the material properties in the pair of sub-layers. The first is to take the larger values of $\varepsilon, \mu$ to be in one sub-layer, i.e. given by Equation (11), and the smaller values in the other sublayer from Equation (10) (case I). For this case, the impedances in the layers are the same, but the refractive index (the square root of the product of the relative $\varepsilon, \mu$ ), suffers from strong jumps at the successive layers. The other possibility is to take the smaller value of $\mu$, Equation (11), together with the larger value of $\varepsilon$, Equation (10), in one sub-layer, and the opposite combination in the other sub-layer (case II) [20]. In this case, the impedances of the successive layers suffer from jumps, but the refractive index profile changes continuously.

\section{Scattering from a Dielectric Sphere with Multi-Layered Coating}

The configuration for electromagnetic scattering by a dielectric sphere coated by $2 M$ layers is shown in Figure 2. The external radius, permittivity, and permeability of the core and the layers are denoted by $a_{i}, \varepsilon_{i}$ and $\mu_{\mathrm{i}}(i=$ $1,2, \cdots, 2 M+1)$, respectively. Figure 2 shows an $E_{x}$ polarized plane wave with amplitude $E_{0}, E^{i}=E_{o} e^{-j K_{0} z} \bar{x}$, incident upon the coated sphere along the $\hat{z}$ direction. $k_{0}=\omega \sqrt{\mu_{0} \varepsilon_{0}}$ is the wave number in free space. The time dependence $e^{j \omega t}$ is suppressed.

The fields in the different regions are expanded in terms of spherical harmonics of $\mathrm{TE}_{\mathrm{r}}$ and $\mathrm{TM}_{\mathrm{r}}$ modes w.r.t. the radial directions. The field $(\mathrm{E}$ or $\mathrm{H})$ with only transverse components $(\theta, \varnothing)$ is expressed by the harmonics $m$, whereas the other field having the three components is expressed by the harmonics $n[25,26]$.

$$
\begin{aligned}
m_{e}^{o}= & \pm \frac{1}{\sin \theta} J_{n}(k r) P_{n}^{1}(\cos \theta) \sin _{\cos } \varnothing \hat{\theta} \\
& -J_{n}(k r) \frac{\partial P_{n}^{1} \sin \theta \hat{\cos } \varnothing \hat{\varnothing}}{n_{e}^{o}=} \frac{n(n+1)}{k r} J_{n}(k r) P_{n}^{1}(\cos \theta) \cos _{\sin } \varnothing \hat{r} \\
+ & \frac{1}{k r}\left[k r J_{n}(k r)\right]^{\prime} \frac{\partial P_{n}^{1}}{\partial \theta} \sin \cos \varnothing \hat{\theta} \\
& +\frac{1}{k r \sin \theta}\left[k r J_{n}(k r)\right]^{\prime} P_{n}^{1}(\cos \theta) \sin _{\cos } \varnothing \hat{\varnothing}
\end{aligned}
$$

where $J_{n}(k r)$ is a spherical Bessel function of order $\mathrm{n}$ of any kind and $P_{n}^{1}(\cos \theta)$ is a Legendre polynomial of the first kind, first order and $n^{\text {th }}$ degree. The $\varnothing$-dependnce is taken as $\sin \varnothing$ or $\cos \varnothing$ (odd, $o$, and even, $e$ ) to conform with the incident plane wave.

The incident plane wave can be expressed in terms of spherical harmonics with Bessel functions of the first kind as:

$$
\begin{gathered}
E^{i}=E_{o} e^{-j K_{0} z} \bar{x}=\sum_{n=1} G_{n}\left(m_{o}^{i}+j n_{e}^{i}\right) \\
H^{i}=-\sum_{n=1} \frac{G_{n}}{Z_{0}}\left(m_{e}^{i}-j n_{o}^{i}\right)
\end{gathered}
$$

where

$$
G_{n}=(-j)^{n} \frac{2 n+1}{n(n+1)} E_{o}
$$

and $Z_{0}=\sqrt{\mu_{0} / \varepsilon_{0}}, j=\sqrt{-1}$.

The scattered fields can be expanded in terms of spherical harmonics with spherical Hankel functions $h_{n}^{2}\left(k_{0} r\right)$ representing scattered outgoing waves with unknown coefficients $e^{s}, d^{s}$ of the TE, TM parts, respectively.

$$
\begin{aligned}
E^{s} & =\sum_{n=1} G_{n}\left(e^{s} m_{o}^{s H}+j d^{s} n_{e}^{s H}\right) \\
H^{s} & =-\sum_{n=1} \frac{G_{n}}{Z_{0}}\left(d^{s} m_{e}^{s H}-j e^{s} n_{o}^{s H}\right)
\end{aligned}
$$

We can write the fields in the $i^{\text {th }}$ Layer as:

$$
\begin{array}{r}
G_{n}\left(d^{c H^{1} i} m_{o}^{c H^{1}}+j e^{c H^{1} i} n_{e}^{c H^{1}}\right. \\
\left.+d_{n=1}^{c H^{2} i} m_{o}^{c H^{2}}+j e^{c H^{2} i} n_{e}^{c H^{2}}\right)
\end{array}
$$




$$
H^{c i}=-\sum_{n=1} \frac{G_{n}}{Z_{0}}\left(\begin{array}{l}
e^{c H H^{1} i} m_{e}^{c H^{1}}-j d^{c H^{1}{ }_{i}} n_{0}^{c H^{1}} \\
+e^{c H^{2} i} m_{e}^{c H^{2}}-j d^{c H^{2 i}} n_{o}^{c H^{2}}
\end{array}\right)
$$

where the modes in the cloak region are represented by Hankel functions of the first and second kinds with arguments $\left(k_{i} r_{i}\right)$, where $k_{i}=\omega \sqrt{\mu_{i} \in_{i}}$, and unknown coefficients $\mathrm{d}$, e. The boundary conditions at the interface between layers $i, i+1$ leads to two equations relating the TM coefficients $d^{c H^{1}}, d^{c H^{2}}$ of the two layers, and two equations relating the TE coefficients $e^{c H^{1}}, e^{c H^{2}}$ of the two layers. The finiteness of the field in the dielectric core leads to the following ratios in the dielectric core [27]:

$$
\frac{d^{c H^{2}(1)}}{d^{c H^{1}(1)}}=1, \frac{e^{c H^{2}(1)}}{e^{c H^{1}(1)}}=1
$$

The ratios $d^{c H^{2}(i)} / d^{c H^{1}(i)}$ and $e^{c H^{2}(i)} / e^{c H^{1}(i)}$ in the successive larger layers can be obtained iteratively from the following equations [27]:

$$
\begin{aligned}
& \frac{d^{c H^{2}(i+1)}}{d^{c H^{1}(i+1)}}=-\frac{H_{n}^{1}\left(K_{i+1} r_{i}\right)-R_{H}^{i} H_{n}^{1^{\prime}}\left(K_{i+1} r_{i}\right)}{H_{n}^{2}\left(K_{i+1} r_{i}\right)-R_{H}^{i} H_{n}^{2^{\prime}}\left(K_{i+1} r_{i}\right)}, \\
& i=1,2, \cdots 2 M \\
& \frac{e^{c H^{2}(i+1)}}{e^{c H^{1}(i+1)}}=-\frac{H_{n}^{1}\left(K_{i+1} r_{i}\right)-R_{E}^{i} H_{n}^{1^{\prime}}\left(K_{i+1} r_{i}\right)}{H_{n}^{2}\left(K_{i+1} r_{i}\right)-R_{E}^{i} H_{n}^{2^{\prime}}\left(K_{i+1} r_{i}\right)}, \\
& i=1,2, \cdots 2 M
\end{aligned}
$$

where $H_{n}^{1}(K R)=K R h_{n}^{1}(K R)$ and $H_{n}^{2}(K R)=K R h_{n}^{2}(K R)$ are the Riccati-Hankel functions.

$$
\begin{aligned}
& R_{H}^{i}=\sqrt{\frac{\mu_{i+1} \epsilon_{i}}{\epsilon_{i+1} \mu_{i}}} \frac{H_{n}^{1}\left(K_{i} r_{i}\right)+\frac{d^{c H^{2}(i)}}{d^{c H^{1}(i)}} H_{n}^{2}\left(K_{i} r_{i}\right)}{H_{n}^{1^{\prime}}\left(K_{i} r_{i}\right)+\frac{d^{c H^{2}(i)}}{d^{c H^{1}(i)}} H_{n}^{2^{\prime}}\left(K_{i} r_{i}\right)}, \\
& i=1,2, \cdots 2 M \\
& R_{E}^{i}=\sqrt{\frac{\mu_{i} \epsilon_{i+1}}{\epsilon_{i} \mu_{i+1}}} \frac{H_{n}^{1}\left(K_{i} r_{i}\right)+\frac{e^{c H^{2}(i)}}{e^{c H^{1}(i)}} H_{n}^{1^{\prime}}\left(K_{i} r_{i}\right)+\frac{e^{c H^{2}(i)}}{e^{c H^{1}(i)}} H_{n}^{2^{\prime}}\left(K_{i} r_{i}\right)}{i=1,2, \cdots 2 M}
\end{aligned}
$$

Finally, the boundary conditions between the outer layer and air lead to the scattering coefficients $b_{n}=$ $d^{s}$ (TM part) and $a_{n}=e^{s}$ (TE part):

$$
b_{n}=-\frac{j_{n}\left(K_{0} R_{2}\right)-R_{H}^{2 M+1} j_{n}^{\prime}\left(K_{0} R_{2}\right)}{H_{n}^{2}\left(K_{0} R_{2}\right)-R_{H}^{2 M+1} H_{n}^{2^{\prime}}\left(K_{0} R_{2}\right)}
$$

$$
a_{n}=-\frac{j_{n}\left(K_{0} R_{2}\right)-R_{E}^{2 M+1} j_{n}^{\prime}\left(K_{0} R_{2}\right)}{H_{n}^{2}\left(K_{0} R_{2}\right)-R_{E}^{2 M+1} H_{n}^{2^{\prime}}\left(K_{0} R_{2}\right)}
$$

The scattering cross section $\sigma_{s}$ and the normalized radar cross sections $Q_{(\theta, \varphi)}$ are given by $[28,29]$ :

$$
\begin{gathered}
\sigma_{s}=4 \pi r^{2} \frac{\left|E^{s}\right|^{2}}{\left|E^{i}\right|^{2}} \\
Q_{(\theta, \varphi)}=\frac{\sigma_{s}}{\pi R_{1}^{2}}=\frac{4}{\left(K_{0} R_{1}\right)^{2}}\left(\left|S_{1}(\theta)\right|^{2} \sin ^{2} \varphi+\left|S_{2}(\theta)\right|^{2} \cos ^{2} \varphi\right)
\end{gathered}
$$

where $S_{1}(\theta)$ and $S_{2}(\theta)$ are defined by:

$$
\begin{aligned}
& S_{1}(\theta)=\sum_{n} \frac{(2 n+1)}{n(n+1)}\left[a_{n} \pi_{n}(\theta)+b_{n} \tau_{n}(\theta)\right] \\
& S_{2}(\theta)=\sum_{n} \frac{(2 n+1)}{n(n+1)}\left[b_{n} \pi_{n}(\theta)+a_{n} \tau_{n}(\theta)\right]
\end{aligned}
$$

In the above two equations $\pi_{n}(\theta)$ and $\tau_{n}(\theta)$ describe the angular scattering patterns of the spherical harmonics used to describe $S_{1}$ and $S_{2}$ and follow from the recurrence relations $[29,30]$ :

$$
\begin{gathered}
\pi_{n}=\frac{P_{n}^{1}(\cos \theta)}{\sin \theta}=\frac{2 n-1}{n-1} \cos \theta \cdot \pi_{n-1}-\frac{n}{n-1} \pi_{n-2} \\
\tau_{n}=\frac{d P_{n}^{1}(\cos \theta)}{d \theta}=n \cdot \cos \theta \cdot \pi_{n}-(n+1) \pi_{n-1}
\end{gathered}
$$

starting with the initial values: $\pi_{0}=0, \pi_{1}=1$.

The total scattering normalized cross section $Q_{s c a}$ follows from the integration of the scattered power over all directions $\theta, \varphi$, given by [31]:

$$
Q_{s c a}=\frac{2}{\left(K_{0} R_{1}\right)^{2}} \sum_{n=1}^{\infty}(2 n+1)\left(\left|a_{n}\right|^{2}+\left|b_{n}\right|^{2}\right)
$$

The backscattering normalized radar cross section $Q_{b}$, applicable to monostatic radar, is given by [31]:

$$
Q_{b}=\frac{1}{\left(K_{0} R_{1}\right)^{2}}\left|\sum_{n=1}^{\infty}(-1)^{n}(2 n+1)\left(a_{n}-b_{n}\right)\right|^{2}
$$

The mode series is truncated at the mode number $n_{m}=$ $K_{0} R_{2}+4 \times\left(K_{0} R_{2}\right)^{1 / 3}+2[29,30]$.

\section{Results}

To check the above analysis, the scattering pattern and the total scattering cross section are calculated for scattering by a dielectric sphere and compared with References $[27,32]$, and for a lossy dielectric sphere coated by a lossy dielectric layer and compared with Reference [28], leading to identical results. 
The scattering properties of cloaked dielectric spheres $\left(\varepsilon_{d}=2,5\right)$ coated with isotropic homogenous layers are investigated concerning the normalized total scattering cross section versus the normalized frequency $\mathrm{k}_{0}$ and for the bistatic radar cross section (RCS) with varying the reduced radius $\mathrm{c}$ and the number of isotropic cloaking layers with $R_{2}=2 R_{1}$.

\subsection{Normalized Total Scattering Cross Section}

For the ideal cloaking case $(c=0)$ the results of the total scattering for dielectric spheres ( for both case I and case II ) with relative permitivities $\varepsilon_{d}=2, \varepsilon_{d}=5$, are found to be greatly identical with the results for a cloaked conducting sphere, shown in Figure $3(\varphi=0[20,21])$. This can be attributed to the fact that the cloaking properties for ideal cloaking $(c=0)$ is independent of the properties of the cloaked object (conductor or dielectric), since the incident field cannot penetrate to the cloaked object [23].

On the other hand, for approximate cloaking $(c \neq 0)$ the cloaked body is not transformed to a point (as in ideal cloaking) but to a finite sphere. For a conducting sphere with $c=R_{1} / 2$ the variation of the total scattering with frequency takes nearly the shape of the scattering by a conducting sphere but with reduced level as shown in Figure 3 [21]. As $c$ decreases $\left(c=R_{1} / 5, R_{1} / 10, R_{1} / 40\right.$, the scattering approaches that of the ideal case $(c=0))$.

For the ideal profile $(c=0), \varepsilon_{r} / \varepsilon_{0}=0$ at $r=R_{1}$, Equation (5). As c increases, $\varepsilon_{r} \varepsilon_{0}$ at $r=R_{1}$ increases. For $c=R_{1} / 40$, $\varepsilon_{r} / \varepsilon_{0} \cong 1 / 3200$. For $c=R_{1} / 10, \varepsilon_{r} / \varepsilon_{0} \cong 1 / 200$.

Figures 4 and 5 show the effect of the reduced radius $c$ on the normalized total scattering cross section versus frequency for cloaked dielectric spheres with relative permitivities $\varepsilon_{d}=2$ and $\varepsilon_{d}=5$, respectively, for case II with $2 M=40$. Compared with the cloaking for a conducting sphere, Figure 3, the scattering from a dielectric sphere shows the presence of some peaks, which decrease as $\mathrm{c}$ decreases. Such peaks may be due to the presence of multiple reflections effects in a dielectric sphere, compared with a conducting sphere [28]. The behavior for $\varepsilon_{d}=$ 2 and $\varepsilon_{d}=5$ for $c=R_{1} / 40$ is nearly identical with the conducting sphere, since it approaches the ideal profile, and has variations for $c=R_{1} / 10$, Figures 3-5. As the frequency increases the total scattering increases because the ratio of the layer thickness with respect the wavelength increases. At the lower frequencies, the scattering decreases as $\mathrm{c}$ decreases, which is the most useful region with low scattering. As the frequency increases the cases with larger $\mathrm{c}$ show less scattering, but this region is not useful because the level of scattering is high. This behavior with the variation of $\mathrm{c}$ is due the multiple reflections and the interaction effects [20].

Figures 6 and 7 show the effect of the number of layers

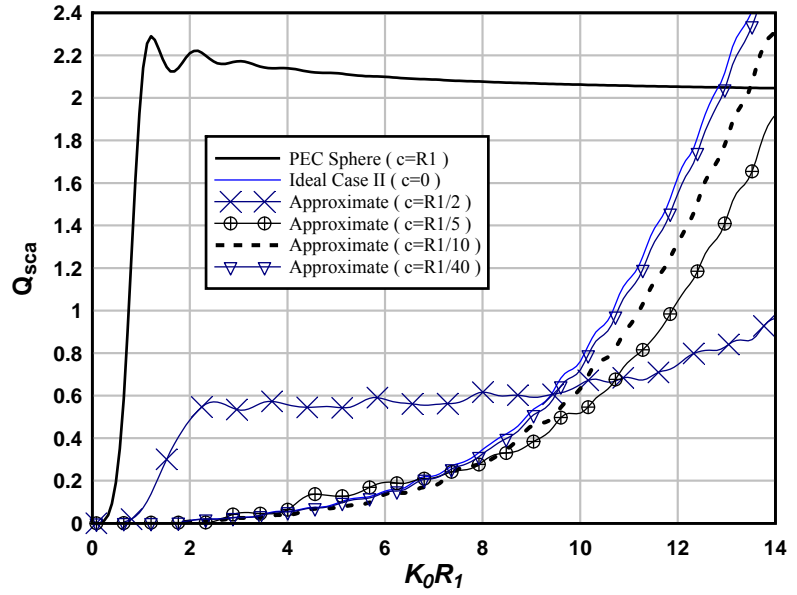

Figure 3. The normalized total scattering cross section of cloaked conducting sphere for different values of $c$ (40Layers) [21].

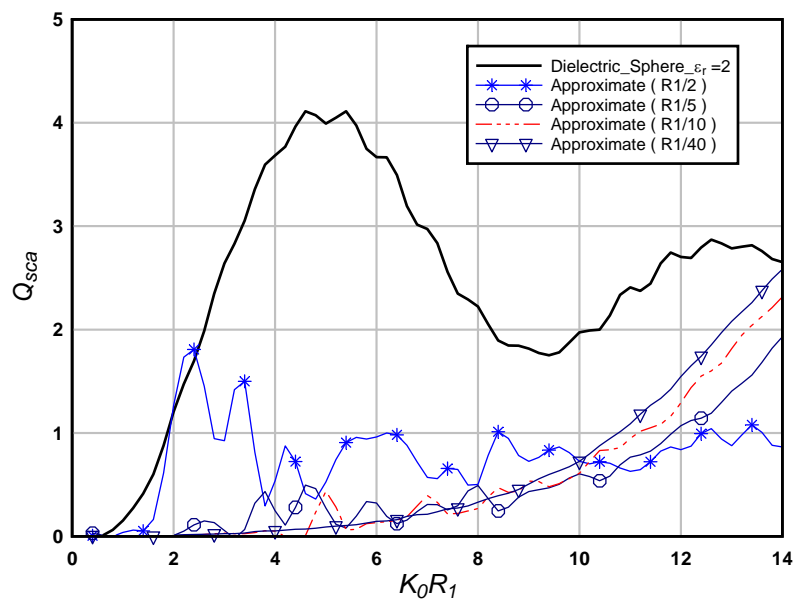

Figure 4. The normalized total scattering cross section of cloaked dielectric sphere $\varepsilon_{d}=2$, with multi-layered isotropic structure for different values of $c$, case II, $2 M=40$.

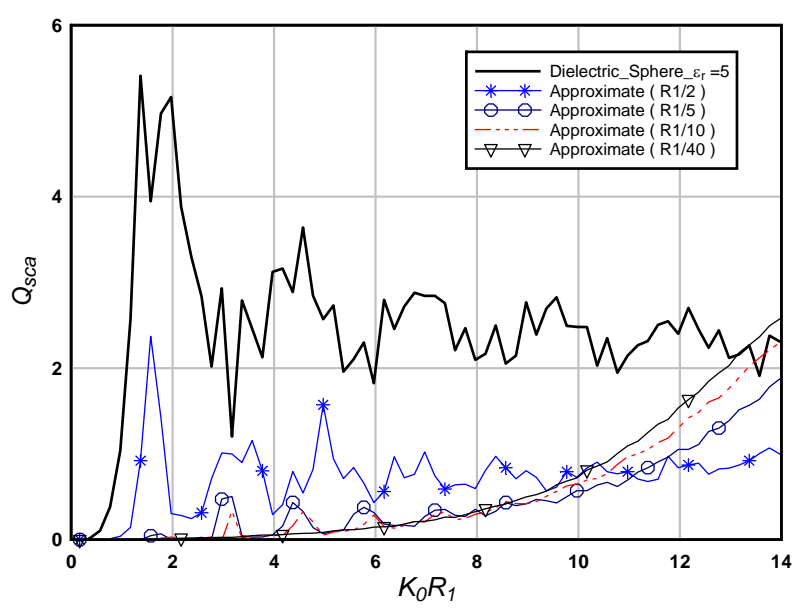

Figure 5. The normalized total scattering cross section of cloaked dielectric sphere $\varepsilon_{d}=5$, for different values of $c$, case II, $2 M=40$. 


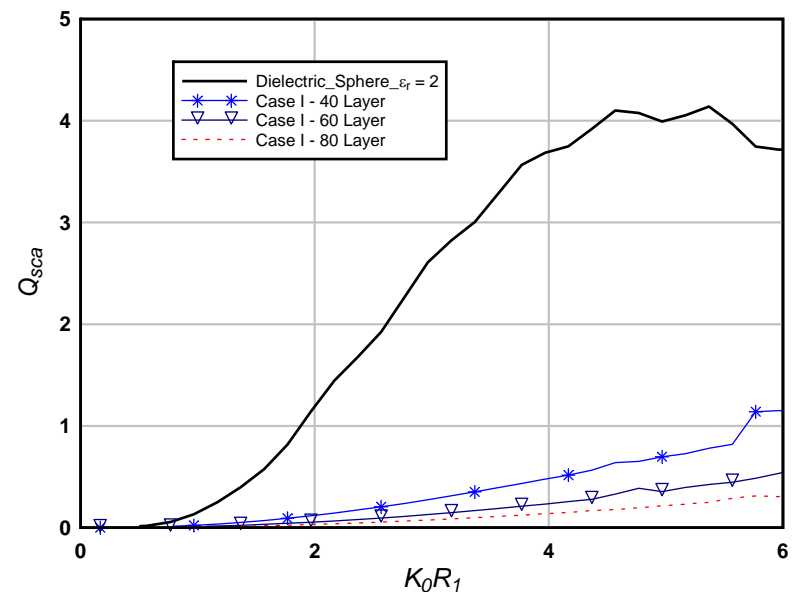

Figure 6. The normalized total scattering cross section for cloaked dielectric sphere with $\varepsilon_{d}=2$ for different numbers of layers, case $I, c=R_{1} / 40$.

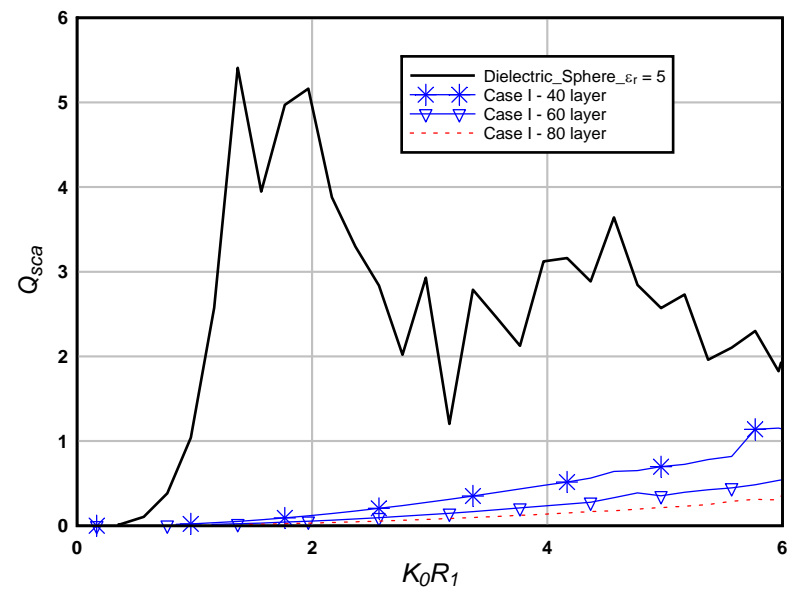

Figure 7. The normalized total scattering cross section for cloaked dielectric sphere with $\varepsilon_{d}=5$ for different numbers of layers, case $I, c=R_{1} / 40$.

on the normalized total scattering cross section versus frequency for case I, for different values of the dielectric constant $\varepsilon_{d}=2$ and $\varepsilon_{d}=5$, respectively, and $c=R_{1} / 40$. The total scattering level is high compared with case II, Figures 4 and 5, since the total scattering results mainly from the forward scattering, which is high for case I, Figures 8 and 9 [20]. The reduction of scattering does not extend beyond $k_{0} R_{1}=6$. As the number of layers increases the scattering decreases. The behavior for $\varepsilon_{d}=2, \varepsilon_{d}=5$ is nearly the same.

\subsection{Bistatic RCS}

Figures 8 and 9 show the effect of the reduced radius $\mathrm{c}$ on the bistatic RCS for $\varepsilon_{d}=2,5$, respectively, for cases I, II, $R_{1}=\lambda$ and $f=2 \mathrm{GHz}$. The scattering pattern for case II for the ideal profile $c=0$ is identical with that for the conducting sphere, Figure 10, and deviates slightly for

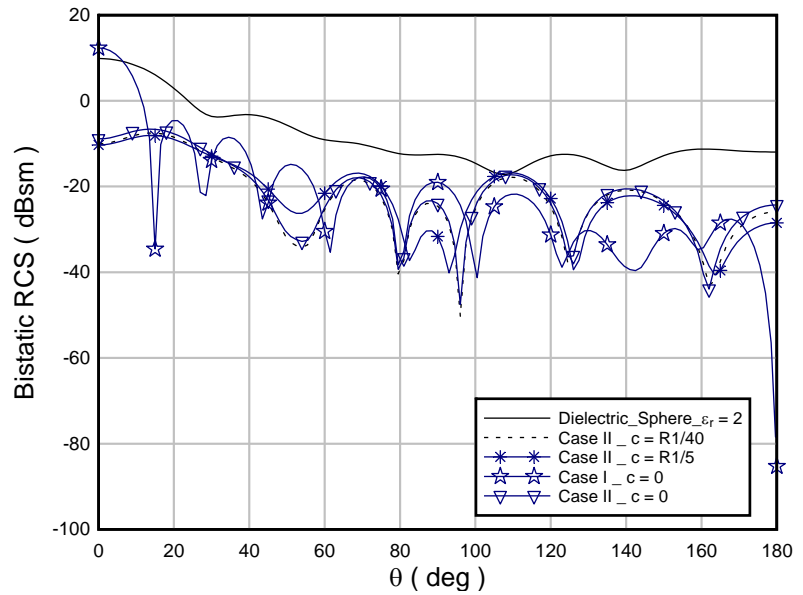

Figure 8. Bistatic RCS of cloaked dielectric sphere with $\varepsilon_{d}=$ 2 for different values of $c, 2 M=40$.

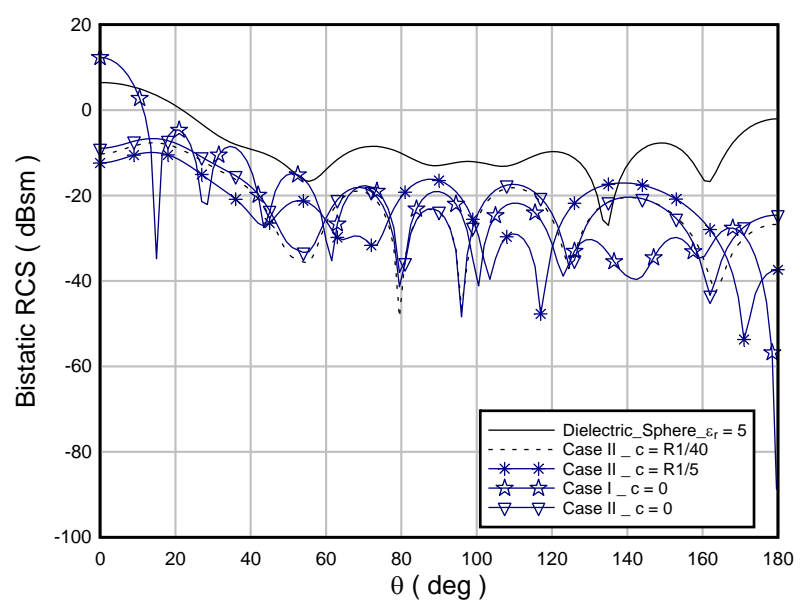

Figure 9. Bistatic RCS of cloaked dielectric sphere with $\varepsilon_{d}=$ 5 for different values of $c, 2 M=40$.

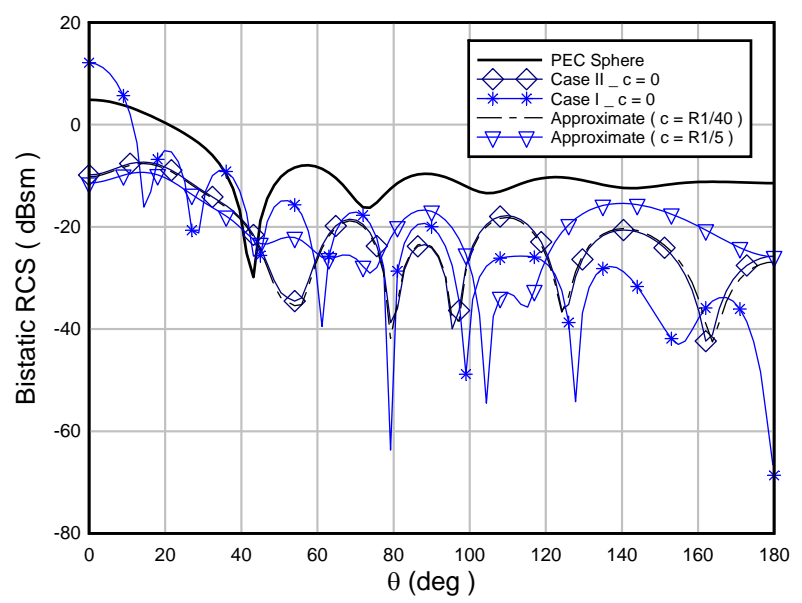

Figure 10. Bistatic RCS of cloaked conducting sphere for different values of $c, 2 M=40$ [21].

$c=R_{1} / 40$ and deviates more for $c=R_{1} / 5$, particularly at the large angles (at and near back scattering, $\theta=180^{\circ}$ ). 
Case I with constant impedance in the layers, shows clear end-fire scattering behavior (forward, $\theta=0^{\circ}$ ) corresponding to phases of radiating elements as the phase of the incident wave [33]. For case I the scattering pattern for ideal cloaking profile deviates from that of the conducting sphere for $\theta>110^{\circ}$. For case II the forward scattering $\left(\theta=0^{\circ}\right)$ is lower than case I [20]. On the other hand the back scattering is lower for case I than case II. As shown in Figures $\mathbf{1 1}$ and $\mathbf{1 2}$ for case I, the pattern for $c=R_{1} / 40$ deviates from the ideal profile also for $\theta>110^{\circ}$.

Figures 13 and 14 show the effect of the number of layers on the scattering patters for case II, for $\varepsilon_{d}=2,5$, respectively, $c=R_{1} / 40$. The results are nearly identical with the conducting sphere [21]. It can be seen that, when the number of layers increases (each isotropic layer is thinner), the scattered field decreases.

\section{Conclusion}

The scattering properties of a cloaked dielectric sphere

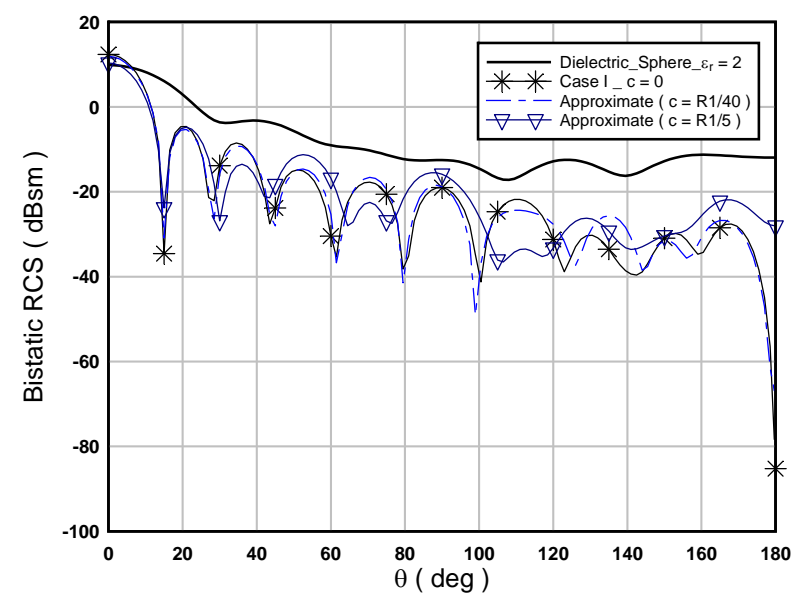

Figure 11. Bistatic RCS of cloaked dielectric sphere for different values of $\mathrm{c}$ for case $\mathrm{I}$ at $\varepsilon_{d}=2,2 M=40$.

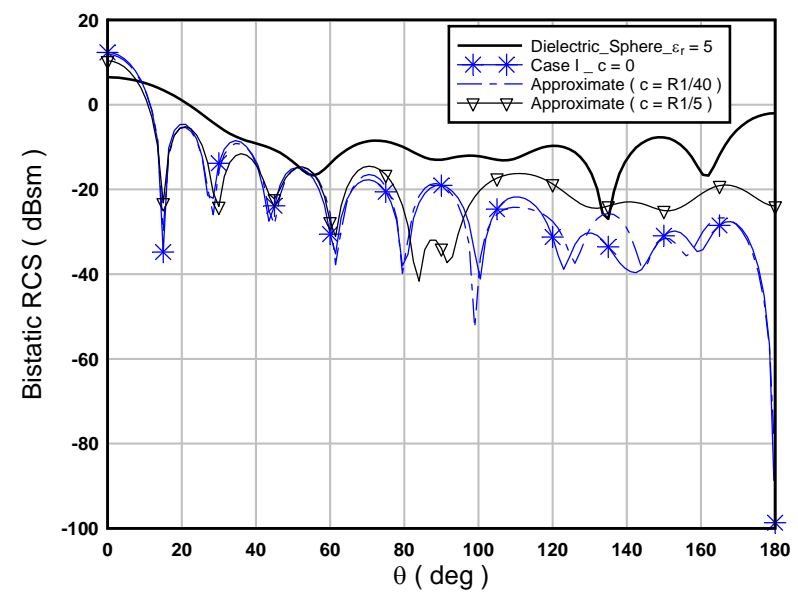

Figure 12. Bistatic RCS of cloaked dielectric sphere for different values of $\mathrm{c}$ for case $\mathrm{I}$ at $\varepsilon_{d}=5$.

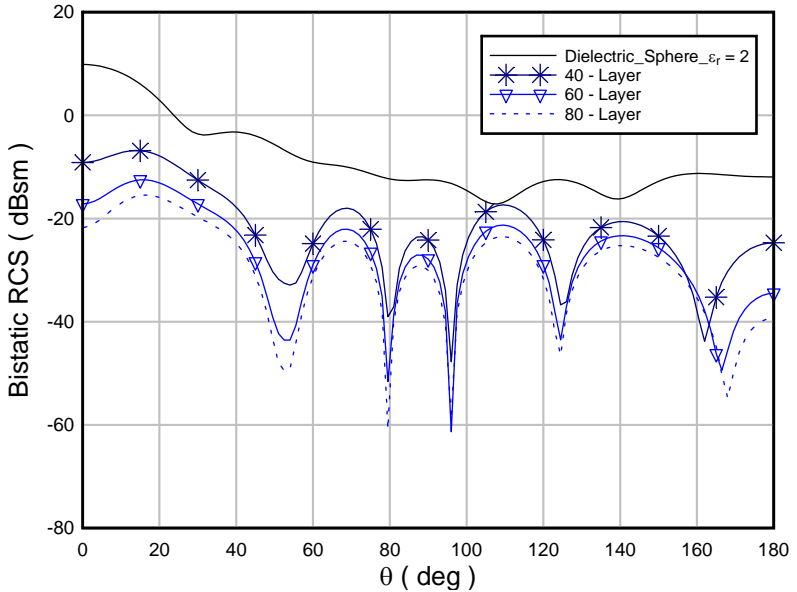

Figure 13. Bistatic RCS for different numbers of layers for case II $\left(\varepsilon_{d}=2\right)$ at $c=R_{1} / 40$.

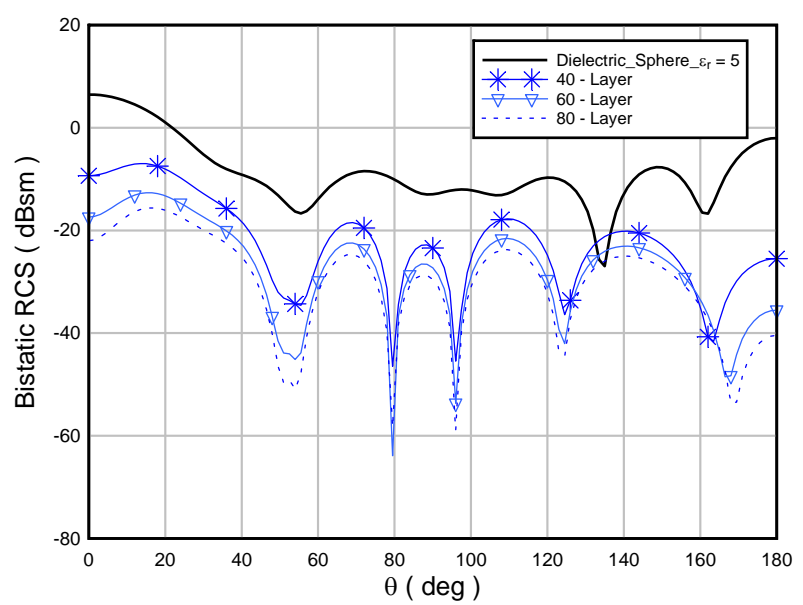

Figure 14. Bistatic RCS for different numbers of layers for case II $\left(\varepsilon_{d}=5\right)$ at $c=R_{1} / 40$.

with isotropic homogenous cloaking layers are investigated concerning the normalized total scattering cross section versus the normalized frequency $k_{0} R_{1}$ and for the bistatic RCS for both case I and case II and with varying the transformed radius, where the dielectric sphere is transformed into a small sphere rather than to a point, together with discretizing the cloaking material using pairs of homogeneous isotropic sub-layers with different numbers. The solution is obtained by rigorously solving Maxwell equations using Mie series expansion. When the number of layers increases (each isotropic layer is thinner), the scattered field decreases. As the transformed radius decreases the total scattering decreases, and approaches the ideal layered cloaking as the transformed radius tends to zero. The discretization has an effect on the scattering at the higher frequencies. The results are compared with the case of cloaked conducting sphere, showing a number of similarities, particularly for small transformed radius. 


\section{REFERENCES}

[1] J. B. Pendry, D. Schurig and D. R. Smith, "Controlling Electromagnetic Fields," Science, Vol. 312, No. 5781, 2006, pp. 1780-1782.

http://dx.doi.org/10.1126/science.1125907

[2] Q. Cheng, W. X. Jiang and T. J. Cui, "Investigations of the Electromagnetic Properties of Three-Dimensional Arbitrarily-Shaped Cloaks," Progress in Electromagnetics Research, Vol. 94, 2009, pp. 105-117. http://dx.doi.org/10.2528/PIER09060705

[3] J. J. Yang, M. Huang, Y. L. Li, T. H. Li and J. Sun, "Reciprocal Invisible Cloak with Homogeneous Metamaterials," Progress in Electromagnetics Research M, Vol. 21, 2011, pp. 105-115. http://dx.doi.org/10.2528/PIERM11090904

[4] A. Shahzad, F. Qasim, S. Ahmed and Q. A. Naqvi, "Cylindrical Invisibility Cloak Incorporating PEMC at Perturbed Void Region," Progress in Electromagnetics Research $M$, Vol. 21, 2011, pp. 61-76. http://dx.doi.org/10.2528/PIERM11061302

[5] X. X. Cheng, H. S. Chen and X. M. Zhang, "Cloaking a Perfectly Conducting Sphere with Rotationally Uniaxial Nihility Media in Monostatic Radar System," Progress in Electromagnetics Research, Vol. 100, 2010, pp. 285-298. http://dx.doi.org/10.2528/PIER09112002

[6] J. Zhang and N. A. Mortensen, "Ultrathin Cylindrical Cloak," Progress in Electromagnetics Research, Vol. 121, 2011, pp. 381-389.

http://dx.doi.org/10.2528/PIER11091205

[7] Y. B. Zhai and T. J. Cui, "Three-Dimensional Axisymmetric Invisibility Cloaks with Arbitrary Shapes in Layered-Medium Background," Progress in Electromagnetics Research B, Vol. 27, 2011, pp. 151-163.

[8] D. Schurig, J. J. Mock, B. J. Justice, S. A. Cummer, J. B. Pendry, A. F. Starr and D. R. Smith, "Metamaterial Electromagnetic Cloak at Microwave Frequencies," Science, Vol. 314, No. 5801, 2006, pp. 977-980.

http://dx.doi.org/10.1126/science.1133628

[9] J. B. Pendry, A. J. Holden, D. J. Robbins and W. J. Stewart, "Magnetism from Conductors and Enhanced Nonlinear Phenomena," IEEE Transactions on Microwave Theory and Techniques, Vol. 47, No. 11, 1999, pp. 20752084. http://dx.doi.org/10.1109/22.798002

[10] G. V. Eleftheriades and K. G. Balmain, "Negative Refraction Metamaterials-Fundamental Principles and Applications," John Wiley, Hoboken, 2005.

[11] N. Engheta and R. W. Ziolkowski, "Metamaterials: Physics and Engineering Explorations," Wiley-IEEE Press, Hoboken, 2006. http://dx.doi.org/10.1002/0471784192

[12] J. Wang, S. Qu, J. Zhang, H. Ma, Y. Yang, C. Gu, X. Wu and $\mathrm{Z}$. Xu, "A Tunable Left-Handed Metamaterial Based on Modified Broadside-Coupled Split-Ring Resonators," Progress in Electromagnetics Research Letters, Vol. 6, 2009, pp. 35-45. http://dx.doi.org/10.2528/PIERL08120708

[13] H. Liu, "Virtual Reshaping and Invisibility in Obstacle Scattering," Inverse Problems, Vol. 25, No. 4, 2009, pp.

\section{1-10. http://dx.doi.org/10.1088/0266-5611/25/4/045006}

[14] T. Zhou, "Electromagnetic Inverse Problems and Cloaking," Ph. D. Thesis, Washington University, St Louis, 2010.

[15] Y. Huang, Y. Feng and T. Jiang, "Electromagnetic Cloaking by Layered Structure of Homogenous Isotropic Materials," Optics Express, Vol. 15, No. 18, 2007, pp. $1-4$.

[16] C. Qiu, L. Hu and S. Zouhdi, "Isotropic Non-Ideal Cloaks Providing Improved Invisibility by Adaptive Segmentation and Optimal Refractive Index Profile from Ordering Isotropic Materials," Optics Express, Vol. 18, No. 14, 2010, pp. 14950-14959.

http://dx.doi.org/10.1364/OE.18.014950

[17] C. Simovski and S. He, "Frequency Range and Explicit Expressions for Negative Permittivity and Permeability for an Isotropic Medium Formed by a Lattice of Perfectly Conducting $\Omega$ Particles," Physics Letters A, Vol. 311, No. 2-3, 2003, pp. 254-263. http://dx.doi.org/10.1016/S0375-9601(03)00494-8

[18] C. Simovski and B. Sauviac, "Toward Creating Isotropic Microwave Composites with Negative Refraction," Radio Science, Vol. 39, No. 2, 2004, pp. 1-18.

[19] C. W. Qiu, L. Hu, X. Xu and Y. Feng, "Spherical Cloaking with Homogenous Isotropic Multilayered Structures," Physical Review E, Vol. 79, 2009, pp. 1-4.

[20] C. M. Ji, P. Y. Mao and F. D. Ning, "An Improved Method of Designing Multilayered Spherical Cloak for Electromagnetic Invisibility," Chinese Physics Letters, Vol. 27, No. 3, 2010, pp. 1-4.

[21] H. Zamel, E. El-Diwany and H. El-Hennawy, “Approximate Electromagnetic Cloaking of a Conducting Sphere using Homogeneous Isotropic Multi-Layered Materials," 2nd Middle East Conference on Antennas and Propagation, 29-31 December 2012, Cairo.

[22] W. Song, X. Yang and X. Sheng, "Scattering Characteristic of 2-d Imperfect Cloaks with Layered Isotropic Materials," IEEE Antennas and Wireless Propagation Letters, Vol. 11, 2012, pp. 53-56. http://dx.doi.org/10.1109/LAWP.2011.2182590

[23] M. Yan, W. Yan and M. Qiu, "Invisibility Cloaking by Coordinate Transformation," Progress in Optics, Vol. 52, 2009, pp. 261-304. http://dx.doi.org/10.1016/S0079-6638(08)00006-1

[24] H. Zamel, E. El-Diwany and H. El-Hennawy, "Approximate Electromagnetic Cloaking of Spherical Bodies," 29th National Radio Science Conference (NRSC), Cairo, 10-12 April 2012, pp. 19-28. http://dx.doi.org/10.1109/NRSC.2012.6208502

[25] J. A. Stratton, "Electromagnetic Theory," McGraw-Hill, Boston, 1941.

[26] R. F. Harrington, "Time Harmonic Electromagnetic Fields," McGraw-Hill, Boston, 1961.

[27] J. Jin, "Theory and Computation of Electromagnetic Fields," John Wiley, Hoboken, 2010. http://dx.doi.org/10.1002/9780470874257

[28] G. T. Ruck, D. E. Barrick, W. D. Stuart and C. K. Krich- 
baum, "Radar Cross Section Handbook," Kluwer Academic, Boston, 1970.

[29] O. Pena and U. Pal, "Scattering of Electromagnetic Radiation by a Multilayered Sphere," Computer Physics Communications, Vol. 180, No. 11, 2009, pp. 2348-2354. http://dx.doi.org/10.1016/j.cpc.2009.07.010

[30] L. Kai and P. Massoli, "Scattering of Electromagnetic Plane Waves by Radially Inhomogeneous Spheres: A Finely Stratified Sphere Model," Applied Optics, Vol. 33, No. 3, 1994, pp. 501-511. http://dx.doi.org/10.1364/AO.33.000501

[31] A. Aden and M. Kerker, "Scattering of Electromagnetic
Waves from Two Concentric Spheres," Journal of Applied Physics, Vol. 22, No. 10, 1951, pp. 1242-1246. http://dx.doi.org/10.1063/1.1699834

[32] N. Tsitsas and C. Athanasiadis, "On the Scattering of Spherical Electromagnetic Waves by a Layered Sphere," Journal of Applied Mathematics and Mechanics, Vol. 59, No. 1, 2005, pp. 55-74. http://dx.doi.org/10.1093/qjmam/hbi031

[33] E. Jordan and K. Balmain, "Electromagnetic Waves and Radiating Systems," Prentice-Hall, Upper Saddle River, 1968. 